XX.

Aus dem pathologischen Institut der Universität Kiel.

\title{
Ueber die Beziehungen der sogenannten Karzinoide des Darms zu den Darmkrebsen.
}

\author{
von \\ Dr. F. Hagemann (Uelzen). \\ (Hierzu Tafel XI und 5 Textfiguren.)
}

In den letzten Jahrzehnten hat unter den Arbeiten iiber Krebs ein gewisser Darmtumor, das „Karzinoid“, eine besondere Rolle gespielt. Schon in seinem Namen driickt sich die Unsicherheit aus, die hinsichtlich seiner Einordnung noch immer besteht. Und doch wäre Klarheit gerade hier besonders wichtig. Es sind verschiedene Gründe, die der besonderen Aufmerksamkejt für diesen Tumor ihre Berechtigung rerleihen. Ein Teil des Verdauungschlauchs ist ein besonders bevorzugter Boden für die Entwicklung von Krebs .... nach Küttner sind $40-45$ pCt. aller Krebsfälle allein im Magen lokalisiert und hier von außerordentlich verschiedener Bösartigkeit. Im Dünndarm dagegen kommt der Krebs recht selten, dagegen häufiger ein krebsähnlicher Tumor vor. Die Größe und Halignität des Karzinoids ist sehr gering, zwei Punkte, die das Studium seines anatomischen Aufbaues sehr erleichtern, ebenso wie sein freies, zum Teil oberflächliches Wachstum. Ferner ist der Umstand, dal man diesen Tumor vermöge seiner Lage häufiger als die meisten anderen in seiner frühesten Entwicklung finden und untersuchen kann, von erheblicher Wichtigkeit.

Die Ansichten in der Literatur iiber das Karzinoid, schon ob es ein echtes Gewächs oder eine Gewebsmißbildung ist, ob es sich um ein Gebilde epithelialen oder endothelialen Charakters handelt, sind sehr geteilt.

Es sei mir gestattet, in folgendén eine Lebersicht der wichtigsten Anschauungen über das Wesen des Karzinoids in Kürze wiederzugeben. um danı zu meinen neuen Fällen überzugehen.

Echte Karzinome des Dïnndarms (0. Lubarsch. 1888. Virch. Arch. Bd. 61, I).

Den Nebenpankreasanlagen nahestehende Fehlbildungen (Oberndorfer.

Frankf. Zeitschr. f. Path. Bd. 1. H. 3/4).

Drïsenpolypen (Langhans. Virch. Arch. Bd. 38. S. 559). 
Hagema n n, Beziehungen d. sog. Karzinoide d. Darms zu d. Darmkrebsen. 405

Epitheliale Tumoren der Dünndarmsubmukosa (E. Tönnießen. 1909. Bd. 8. S. 355. - 11 Fälle)

Er betont ihr nur expansives Wachstum und will kein infiltratives Wachstum beobachtet haben. Er faßt die Tumoren auf als „ein Produkt der Fähigkeit des Dünndarmepithels zur Sprossung und Drüsenbildung“. Es sei in der Literatur kein einziger einwandfreier Fall von Dünndarmkarzinom $\mathrm{zu}$ finden.

Den Hautnaevis gleichzustellende Gebilde (Aschoff. Münch. med. Wochschr. 1910. S. 1914).

Saltykow. (XV. Tagung d. deutsch. path. Gesellsch. z. Straßburg i. E., April 1912), der die Dünndarmkarzinoide mit den Langerhans'schen Inseln des Pankreas in Verbindung bringt - „Pankreaskeime, die Inselgewebe produzieren können" --, meint, daß aus versprengten Pankreaskeimen hervorgehen können:

I. Adenomyome: Kanalsystem vom Typus der Ausführungsgänge,

II. Nebenpankreas: Kanalsystem und herausdifferenzierte Azini,

III. Karzinoide Tumoren: Inselgewebe,

IV. Uebergangsformen der vorigen Gruppen.

Nach Saltykow sprechen folgende Gründe für die Verwandtschaft der karzinoiden Tumoren mit den Langerhans'schen lnseln: die Zellen sind abgerundet oder eckig, die Zellgrenzen verwischt, nur der Kern sichtbar - das Protoplasma unscharf begrenzt, wie im Zerfall, bei van Gieson-Präparaten hell. - Es kommen oft kompakte Synzytiummassen vor. - Die Zellkerne sind vơn groben Chromatinfäden durchsetzt, enthalten mehrere Kernkörperchen, mitunter Riesenkerne. Das sei alles typisch für die Zellen der Langerhans'schen Inseln. Gegen das Wesen der Karzinoide als echte Karzinome sprechen aber nach Sal tykow folgende Punkte:

1. Die größeren Zellkomplexe sind von einem mehr oder weniger dichten Netz von Kapillaren und kleinen Gefäßen durchsetzt - manchmal fallen die Bindegewebssepten mehr auf als die darin enthaltenen Gefäße (auch Lubarsch und Tönnießen sprechen von netzförmig durchbrochenen Zellnestern). Sonst sei es für Krebs gerade charakteristisch, daß innerhalb der Alveolen Gefäße und Bindegewebe fehlen:.

2. Die Zylinderzellen können nicht mit den Zellen der Karzinomzellschläuche verwechselt werden, da ihre Form anders und ihr Kern einerseits nicht an der Basis, sondern in der Mitte oder nach dem freien Rande zu liege, andererseits nicht oval, sondern in der Regel rund ist; der dem Bindegewebe aufsitzende Protoplasmasaum ist manchmal auffällig breit, außerdem liegen im Zentrum der Zylinderschläuche meist ein Gefäß, kein Drüsenlumen.

Er führt von 7 karzinoiden Tumoren 5 im Dünndarm, je 1 im Rektum und Flexura sigmoidea an; 5 Adenomyome, alle im Dünndarm. 
406 Hageman $\mathrm{n}$, Beziehungen d, sog. Karzinoide d. Darms zu d. Darmkrebsen.

Versè (Arbeiten aus d. Path. Inst. z. Leipzig. Bd. 1. H. 5) beschreibt 9 lälle. Er hält sie für echte Karzinome mit dentlich infiltrativem aber relativ gutartigem Wachstum, weil sie a) szirrhös, b) keinen mechanischen Reizen ausgesetzt seien. Im Gegensatz zu Saltykow fand er sie fast nur multipel.

Burekhard (Frankf. Zeitschr. 1. Path. Bd. 3. H. 3 u. Bd. 11. H. 2/3) beschreibt zweimal 6 eigene Fäle als Karzinome. Thre Besonderheiten sind:

1. Ihr häufiges multiples Auftreten.

2. Die Zellen sind auffällig klein.

3. Sie bilden meist solide Stränge von kleinen rundlichen Zellen.

4. Ihre "organartige": Regelmäßigkeit, besonders Dickdarm- und Magenkarzinom gegenüber.

5. Klinisch kommen sie meist bei älteren Leuten vor über dem durchschnittlichen Karzinom-Alter.

6. Sie haben infiltratives, aber relativ beschränktes Wachstum, geringe Neigung zu Ulzeration and Netastasierung.

7. Häufig hängen die Stränge mit Lieberkühn schen Krypten zusammen. - Also Entstehung durch Wucherung des Drüsenepithels! Für ihren karzinomatösen Charakter führt er an:

1. den netzförmigen Zusammenhang der soliden epithelialen Stränge,

2. das inflitrative Wachstum bei allen größeren Tumoren,

3. die Metastasenbildung:

Die Tumoren zeigen kein Zeichen eines progredienten Wachstums, es fehlt meist eine Reaktionszone am Rand, doch sind auch wiederholt Lymphozyteninfiltrate und leichte Wucherung der Lymphgefäliendothelien in der Tumorumgebung. gefunden. Gegen ihren karzinomatösen Charakter sprechen nach Burckhard:

1. ihr regelmäßiger Bau, der aber trotzdem malignes Wachstum zeigen kann,

2. ihre relative Gutartigkeit,

3. bei den Appendixkarzinoiden das jugendliche Alter der Befallenen,

4. histologisch: die Kleinheit und Gleichförmigkeit der einzelnen Zellen.

Bei seinen späteren 6 Fällen hebt Burckhard noch die Unterschiede gegen Fehlbildungen (Pankreaskeime usw.) besonders hervor:

1. in den Fehlbildungen kommen nirgends solide Stränge vor - Pankreasläppchen können nicht verwechselt werden,

2. bei Adenomyomen ist die Muskulatur, welche frei in der Submukosa liegt, ein hauptsächliches Unterscheidungsmerkmal,

3. nirgends findet sich dort infiltratives Wachstum,

4. Pankreasteile, welche die Muskularis durchbrechen, sind stets von Bindegewebe umgeben, 
Hagemann, Beziehungen d. sog. Karzinoide d. Darms zu d. Darmkrebsen. 407

5. es besteht dort kein Zusammenhang mit den Lieberkühn'schen Krypten.

Zum Untersehied gegen andere Darmkarzinome finden sich bei den Karzinoiden:

a) klinisch:

1. nie gröBere ähnliche Tumoren oder zirkuläres Wachstum,

2. nur sehr selten makroskopisch sichtbare Metastasen, Sie sind also relativ gutartig,

3. häufig multiples primäres Wachstum,

b) histologisch:

1. Kleinheit der Zellen,

2. solide Stränge (Carc. solid. simplex).

Burckhard weist noch besonders auf die Aehnlichkeit des Karzinoids mit Krompecher's Basalzellenkrebs hin.

Eine ähnliche Zwiespältigkeit der Meinung liegt vor bei den Appendixkarzinoiden. Da sind es: im Wurmfortsatz: entzïndliche Endotheliome (Milner, D. Zeitschr. f. Chir. Bd. 102. H. 1-3), fibroepitheliale Neubildungen (Goetjes) oder mehr oder weniger echte Krebse (Brieger, Winkler, Simmonds, Kojetzni).

Ich komme jetzt zu der Beschreibung einiger neuer Fälle, gebe erst das Wesentliche des Obduktionsbefundes, dann eine genanere Beschreibung des Karzinoids im mikroskopischen Bilde.

1. Fall: Johann B. (S. N. 710. 1914), 61 Jahre alt, Schrankenwärter. Mächtiger, stark zerfallener Krebs des Magens mit starker Blutung und Durchbrueh in den linken Leberlappen und das Pankreas. Flüssiges und geronnenes Blut im Magen und im Darm. Abszeh, Nekrosen und Blutungen des linken Leberlappens. Nekrosen und Entzündungsherde des Pankreas. Unmittelbares Uebergreifen des Krebses auf Leber, Pankreas und Dickdarmserosa. Verwachsungen zwischen Magen und Leber, Netz und Därmen. Metastasen der retroperitonealen, periportalen und mesenterialen Lymphknoten sowie des rechten Leberlappens. Hochgradige allgemeine Anämie und Abmagerung. Sehr starke Hämosiderose der Milz und Leber. Gallertig atrophisches und rotes hnochenmark. Mäßige Atherosklerose der Bauchaorta und Herzklappea, geringe der Brustaorta, starke der Kranzarterien des Herzens, der Becken- und Beinarterien. Ausgedehnte Schwielenbildung der Herzmuskulatur, zahlreiche Narben besonders der linken Niere. Braune Atrophie der Herzmuskulatur, der Nebennieren, Leber und des Hodens. Linksseitiger Hydrothorax, Knöchelödeme, Emphysem und Anthrakose der Lungen. Oedem der Langenunterlappen. Antbrakose der Lymphknoten des Brust- und oberen Bauchraums. Blutresorption and Hämosiderose einiger perigastrischer Lymphknoten. Atrophie des Schädeldachs. Hydrocephalus internus und externus. Zysten desPlexus chorioideus, Zysten der rechten Niere. Struma subprarenalis. Subseröses Magenmyom. Laparotomienarbe. 3 sogenannte Karzinoide des Dünndarms.

Mikroskopisch: Der Magenlitebs stellenweise ausgesprochen rein adenomatös (große mit einzeiligem hohen Zylinderepithel ausgekleidete Drüsenräume), 
stellenweise ausgesprochen szirrhös (kleine za einer Reihe oder zwei Reihen angeordnete Epithelstränge), von den Lymphknotenmetastasen sind einige rein adenomatös und medullär, andere fast rein szirrbös. Die Dünndarmmetastasen sind solide und medullär, am meisten an sogenannte Karzinoide erinnernd. Im Pankreas z. T. indurierende Entzündung, z. T. Nekrosen und Eiterungen sowie Krebswucherungen, idas Gleiche am linken Leberlappen. Im übrigen in der Leber sehr starke Leberzellenbämosiderose, interlobuläre Entzündung und Eisenpigmentablagerüg im interlobulären Bindegewebe. Sehr starle Pulpa- und Kapselhämosiderose der Milz. In der Niere sind die Narben sehr reich an bämosiderinbaltigen Spindel- und Randzellen (s. auch Taf. XI, Fig. I a u.b). Die 3 Karzinoide finden

Figur 1.

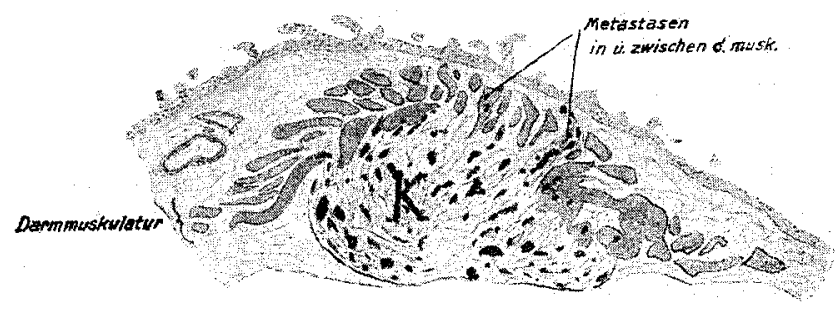

Figur 2.

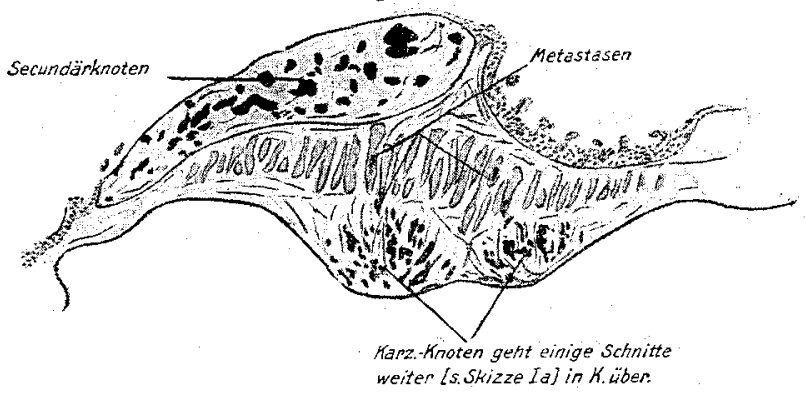

sich im unteren 'Teil des Dünndarms als unter der Schleimhant sich vorwölbende Knoten yon Linsen- bis Kirsobkerngröße, von denen der größte eine schmutziggelblichgrüne Oberfläche besitzt, und, wie sich auf dem Durchschnitt ergibt, z.T. noch ron Schleimhaut überzogen ist. - Mikroskopisch handelt es sich um einen kleinen Knoten, der in der Hauptsache zwischen der Serosa und Muskulatur liegt. In der Grenzschicht zwischen Tumor und Muskulatur sieht man bäufig Tumorzellgruppen und z. T. Stränge, die sich stellenweise bis in die Muskulatur hinein und durch diese hinduroh bis in die Mukosa hinein verfolgen lassen, bier an einer Stelle eine Art Tochtertumor bildend. Die Gesamtmasse des Stroma ist erheblich größer als die des übrigen Gewebes. Letzteres besteht aus dicht gelagerten Zellen, welche zu unregelmäßigen Haufen oder Strängen oder seltener undeutlichen Röhren angeordnet sind. Das Ganze läßt so, wie sich auf Schnittfolgen beobachten läßt, 
einen schwammartigen Aufbau erkennen. Die einzelnen epithelähnlichen Zellen sind klein, haben einen zu ihrem Plasma unverbälthismäfig groben, meist rundlichovalen Kern von starker Färbbarkeit, während der schmale Protoplasmasaum unscharf begrenzt und meist sehr blaß gefärbt ist. Eine Reaktionszone zwischen Tumor und normalem Gewebe ist nicht erkennbar. In dem Stroma finden sich vereinzelt kleine Blutgefäße und Kapillaren.

2. Eall. Magda F. (S. N.824. 1914), 20 Jahre alt, Dienstmädchen. Klinisch war ein Sarkom des linken Oberschenkels festgestellt. Da die Absetzung des Gliedes verweigert wurde, versuchte man eine Radiumbestrahlung. Der Exitus trat wenige Wochen später ein. Die Obduktion ergab: Sarkomatöses Osteoblastom des unteren Femurdrittels links. Riesige Durchblutung und Verjauchung der Weichteile am linken Oberschenkel und der oberen Hälfte des linken Unterschenkels. Blat- und Blutpigmentresorption in den linken inguinalen Lymphknoten. Hochgradige Anämie. Verfettung der Leber. Leichte Sklerose der Aorta. Kleines Jejunumknötchen.

Figur 3.

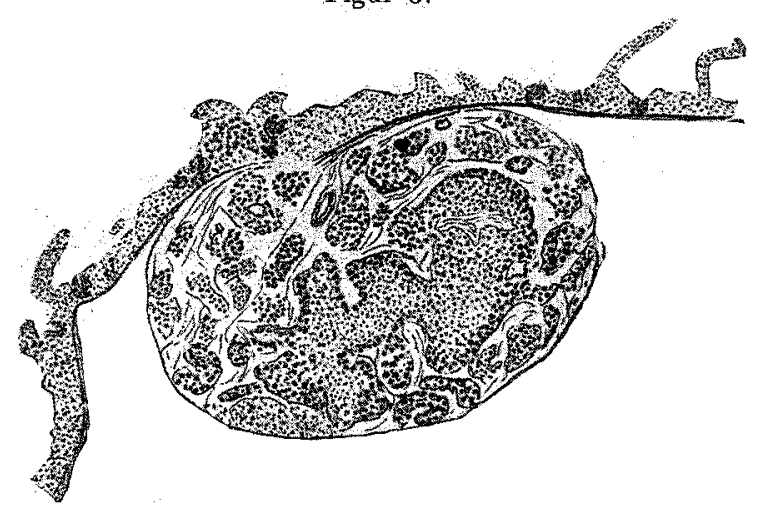

Mikroskopisch: Geschwulst zum größten Teil verjaucht und durchblutet. Nar kleine Bezirke erhaltenes Gewebe. Sebr vielgestaltete Zellen, zahlreiche Riesenzellen, faserige Zwischensubstanz, stellenweise osteoiden Bälkchen ähnelnde Gebilde. Leber: ziemlich starke Verfettung, leichte Staungshyperämie und Oedem. Inguinale Lymphknoten: Füllung der Lymphsinus mit verfetteten spindeligen Zellen. Knochenmark herdweise sehr zellreich. Erythroblasten; polynukleäre Leukozyten (s, auch Taf. XI, Fig. IIa-IIc.)

Das Jejunumknötchen ist etwas über stecknadelkopfgroß, liegt der Hauptsache nach in der Submukosa, doch sind an einer Stelle Zapfen bis in die Mukosa hinein zu vorfolgen. Es unterscheidet sich hier von den übrigen Schleimbautbestandteilen schon durch die Verfettung der Zellen, wie bei Sudanfärbung besonders deutlich hervortritt. Im übrigen ist der Tumor sehr äbnlich dem vorher beschriebenen, nur tritt das Stroma erheblich mebr zurück. Die spithelialen Zellen sind meist zu so großen, unregelmäßigen, soliden Haufen angeordnet; dab ein strang- oder röhrenförmiger Charakter nicht mehr zu erkennen ist. 
3. Fall: Peter M. (S. N. 848. 1915) 63 Jahre alt, Arbeiter.

Klinische Diagnose: Chronische Nephritis, Urämie, Herzdilatation und -insuffizienz. Staungsorgane.

Die Obduktion ergab: Chron. fibröse Peribronchitis der rechten Lunge and der Hilusgegend mit Pleuraverwachsungen. Chron. indurierende Pneumonie und Bronchiektasenbildungen. Fibröse Orchitis und Periorchitis. Chron. indurierende Nephritis. Hochgradige allgemeine Amyloidose. Schinkenmilz, Amyloid der Nebenniere, Nieren, Milz, Leber, Pankreas, der Schilddrüse, des Herzens, des Dänn- und Dickdarms sowie des Magens. Leichte nekrotisierende Entzündung der Dickdarmschleimhaut, eitrige Pelveoperitonitis, Verwachsungen zwischen einer Dünndarmschlinge und dem S romanum, Blutungen der Magen- und Dickdarmschleimhaut. Fibrinöse Gastritis und Oesophagitis. Erosionen der Mastdarmschleimhaut. Pyelitis und Ureteritis cystica beiderseits. Urocystitis cystica. Kleine Papillome und Di-

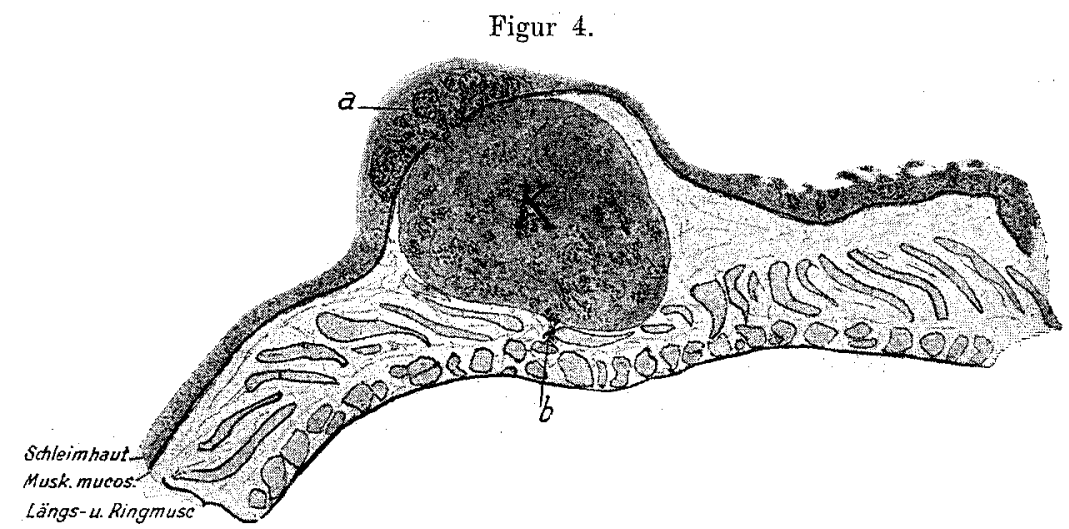

vertikel der Harnblase. Narben der Harnröhre. Verfettung der Herzmuskulatur. Thrombosen der Herzspitze, des Plexus prostaticus und der Vena femoralis rechts. Embolie von Lungenarterienästen, hämorrhagischer Infarkt des linken Lungenunterlappens. Serös-fibrinöse Pleuritis. Eitrige Bronchitis und fibrinöse Pneumonie des rechten Unterlappens. - Atherosklerose der Herzklappen, Kranzarterien, Karotiden, Aorta, der Becken- und Beinarterien. - 3 Karzinoide des Dänndarms (im mittleren Heum findet sich unter der Schleimhaut ein kaum stecknadelkopfgroßer, sich fest anfühlender, leicht hervorragender Herd. Etwas unterhalb davon ein etwas über stecknadelkopfgroßer und gleich daneben ein reiskorngroßer, gleichartiger, auch nach der Serosa sich vorwölbender Herd). Chylusgefäßerweiterungen im Jejunum und Ileum. Herdförmige branne Pigmentierung der Leber. Dystopie der rechten Nebenniere. Psammom and Knochenspangen der harten Hirnhaut. Verwachsungen der Dura und chronisch rezidivierende Pachymeningitis interna haemorrhagica. Blutangen am Boden der linken Gehirnseitenkammer. Traktionsdivertikel der Speiseröhre.

Mikroskopisch: Darm: starkes Zottenamyloid und im Dickdarm der Kapillaren. Starkes Amyloid der Muscularis mucosae und der Muskulatur im Be- 
reiche der Karzinoide. Es handelt sich um Tumoren, deren bindegewebiges Gerüst an Masse das äbrige Gewebe erheblich überragt. Im Bindegewebe finden sich auffällig reichlich Kapillaren und Blutgefäßchen. Das epitheloide Gewebe setzt sich meist aus soliden Zellsträngen und -Haufen zusammen. Die Zellen sind rundlich oder polygonal, ihre Kerne rund; es fällt hier besonders auf, dafb trotz. der im allgemeinen fast gleichen Kerngröße doch vereinzelte bis um das Doppelte größere Zellkerne vorkommen, die dann regelmäßig blasser gefärbt sind. Bei den selten vorliegenden Zellschläuchen liegen in den bier zylindrisch geformten Zellen die Kerne meist im inneren Zelldrittel. Die Tumoren liegen wieder in der Submukosa: Bei den größten Knötchen durchdringt das Tumorgewebe die Muscularis mucosae und hat auf seinem Scheitelpunkt völlig die Schleimhaut verdrängt bzw. ersetzt. An einzelnen Stellen dringt das Tumorgewebe auch in die Ringschicht der Darmmuskulatur ein; hier sieht man die Tumorzellen ohne weitere Umhüllung in den Muskelfasern selbst liegen; deren Zellen hier vielfach keine Kernfärbung mehr aufweisen. Ein kleineres Knötchen wird auf Serienschnitten in seiner ganzen Breite verfolgt. Man kann hier im Verlaufe der Serien gut sehen, wie alle Tumorteile miteinander im Zusammenhang stehen, also vermutlich von einem Zentrum ausgegangen sind. Das gilt sowohl von den kleinen Teilen in der Darmmuskulatur als auch besonders von einem größeren unverkennbaren Tumorteil, der, in der Schleimhaut liegend, in der Hauptsache dem größten rundlichen Tumorteil wie eine Kappe aufsitzt, aber in seinem Zentrum durch einen Stiel aus Tumorgewebe, der die Muscularis mucosae durchbricht, mit dem Hauptteil zusammenbängt und so auf dem Schnitte das Bild eines Pilzes gibt. Von dem in der Schleimhaut liegenden Tumorteil fällt als Besonderes nur auf, daß er im Gegensatz zu dem Hauptknoten fast gar kein Bindegewebe aufzuweisen hat.

4. Fall: Anna P. (S. N. 335. 1916), 30 Jahre alt, Zimmermannsfrau.

Klinische Diagnose: Extraperitoneales Hämatom nach Prolapsoperation.

Die Obduktion ergab: Verletzung der rechten Vena hypogastrica, z. T. zerfallene Thromben der Beckenvenen. Embolische Abszesse und Herde in den Lungen. Subplearale Blutungen und fibrinöse Pleuritis. Pulpaschwellung der Milz. Verfettung der Herzmuskulatur. Staungs- und Fettleber. Kleine Narben der Nieren. Geringe Sklerose der Aorta. Offenes Foramen ovale. Follikuläreitrige Gaumenmandelentzündung links. Zysten der Leber und Niere. Karzinoid des Dünndarms. Es handelt sich um ein kleinerbsgroßes, festes, gestieltes Knötchen. Es liegt der Hauptsache nacb in der Submukosa.

Mikroskopisch: Epithel und Schleimhäute zeigen ziemlich starke Verfettung. Das Stroma ist sehr schwach entwickelt und weist nur selten kleine Gefäßchen auf: Die epithelähnlichen Zellen sind - meist zylinderförmig - zu ein Netzwerk bildenden Schläuchen angeordnet, deren Größe verschieden ist, aber die durchschnittliche Breite der Lieberkübn'schen Krypten um nicht mehr als das Doppelte übertrifft. Man findet unter den die Schlauchwand bildenden Zellen viele, die durch die auffällig breite Basis außen und das sehr stark zugespitzte innere Ende eine keilförmige Gestalt angenommen haben. Dazwischen finden sich vereinzelt Zellen, die einen aufgequollenen, fast blasigen Eindruck machen. Die Zellkerne, rundlich, groß, sind meist im unteren Dritteil, nach der Schlauchauben- 
wand gelagert. Der Inhalt der Schläuche besteht aus Zellen mit sehr großem rundlichen Kern und ganz geringem Protoplasmasaum. Die Zellgrenzen sind hier nicht scharf auseinander zu halten. Die beschriebenen Schläuche durchbrechen an. verschiedenen Stellen die Muscularis mucosae und sind bis tief in die Schleimhaut hin zu verfolgen. Ein direkter Zusammenhang mit den Li eberk üh n'schen Krypten ist hier nicht nachzuweisen. Wohl aber findet sich an einer Stelle eine Krypte, die an ihrem unteren Ende plötzlich za einem längeren soliden Strang wird, dessen Zellen nicht von dener den Inhalt der vorher beschriebenen Schläuche bildenden zu unterscheiden sind. Eine Reaktionszone zwischen Tumor und normalem Gewebe besteht nicht. In der Darmmuskulatur läßt sich der Tumor nicht mehr nachweisen.

5. Fall: Nikolaus F. (S. N. 351. 1916), 66 Jahre alt, Obermaschinist. F. sollte wegen Blasenblutungen operiert werden, starb aber vorher. Die Obduktion

Figur 5.

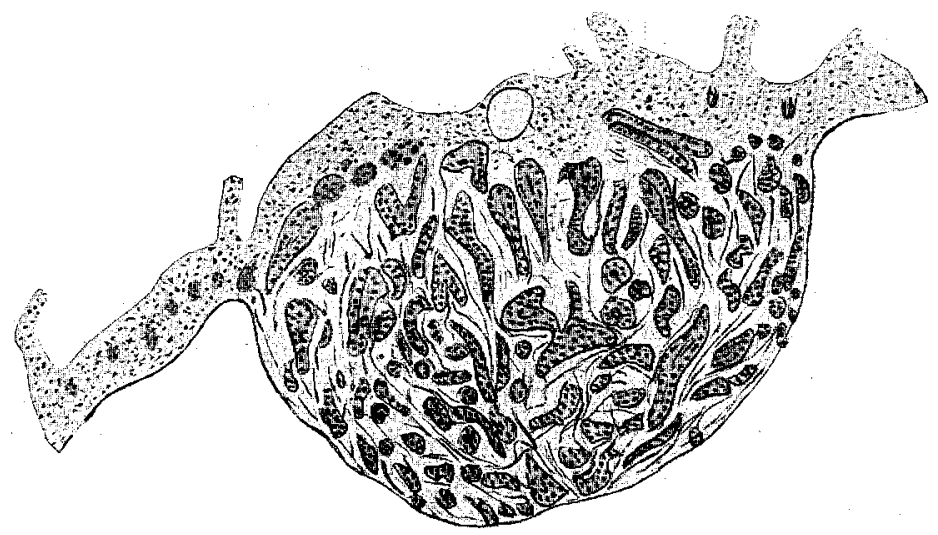

ergab: Multiplo papilläre Epitheliome der Harnblase, schleimiger Katarrh der Blase. Blutergüsse in das Blasenlumen. Starke allgemeine Blutarmut. Schlaffheit und braune Pigmentierung des Herzens. Atrophie der Milz, Nobennieren, Leber, Nieren. Emphysem der Lungen. Verkalkung des Kehlkopfes und der Luftröhrenknorpel. Katarrhalische Bronchitis. Lungenödem. Hydrothorax. Pleuraverwachsungen rechts. Sehnenflecke des Epikards. Alte abgelaufene Aortenendokarditis. Sklerose der Aorta und peripherer Arterien. Traktionsdivertikel der Speiseröhre. Verödung des Wurmfortsatzes. Zysten der Niere. Kolloidzystisches Adenom der Schilddrüse. Karzinoide des Jejunums. Im Jejunum liegt ein blasses, mit gelber Schleimhaut überzogenes Knötchen, ein etwas kleineres ebensolches weiter unten (s. auch Taf. XI, Fig. III).

Mikroskopisch: Die beiden kleinen Tumoren haben in ihrem Aufbau große Aehnlichkeit mit denen der vorher beschriebenen Fälle, doch weisen sie auch wieder kleine Verschiedenheiten auf. Hier sind die Stränge durchweg hohl, sind ziemlich gleichmäßjig stark, ihre Größe schwankt nur wenig um die durchschnittliche Größe der Lieberkühn'schen Krypten. Die Tumorschläuche ziehen bis in 
die Mukosa und sind stellenweise nur schwer ron den Krypten zu unterscheiden. Dié Zellen deutlich zylindrisch, viele haben an ihrem äußeren Rand einen auffällig breiten Faß, der Kern liegt meist etwa in der Mitte der Zellen oder in dem. nach dem Lumen zugekehrten Drittel. Die Schlauchzellen des Tumor weisen. stärkere Verfettung als die Mukosazellen auf.

6. Fall: Fr. (S. N. 517. 1916), 38 Jahre alt, Ehefrau.

Klin ische Diagnose: Placenta praevia.

Die Obduktion ergab: starke allgemeine Blutarmut nach Zerreißung des Uterushalses bei Placenta praevia. Unvollštändiger Zervixriß. Kleiner Einriß an der Portio uteri. Blutige Infiltration des linken Parametriums. Großer puerperaler Uteius, Wahres Corpus lateum rechts. Verfettung der Leber. Follikelschwellung der Milz. Geringe Follikelschwellung im Dickdarm. Rotes Oberschenkelknochenmark. Pleuraverwachsungen. Sehnenflecke des Epikards. Narben und Schwund der Gaumenmandeln. Narben und kleine Zysten der Nieren. Geringe Atherosklerose der Aorta. Abgeheiltes variköses Unterschenkelgeschwür, rechts mit starkem chronischen Ekzem. Oedem des Unterhautzellgewebes. Karzinoide des Ileum. Im oberen llenm, das wie die ganze Darmschleimhaut enorm blaß ist, findet sich ein gut linsengroßer, oberflächlich abgeplatteter Knoten, ebenso weiter unterhalb, oberhalb der Ileozökalklappe. Die Follikel sind hier als stecknadelkoplgroße derbe Knötchen zu erkennen.

Mikroskopisch: Der stark abgeplattete Knoten im unteren lleum zeigt regelmäßig angeordnete solide Zellzapfen mit Durchbrechung der Muscularis mucosae.

7. Fall: Wilhelm K. (S. N. 678. 1916), 70 Jahre alt, Arbeiter.

Klinische Diagnose: Kardiakarzinom.

Die Obduktion ergab: jauchig zerfallener Krebs der unteren Speiseröhre und des Mageneingangs, Einwuchern des Krebses in die rechte Lunge. Metastasen der Lymphknoten an der Kardia. Pleuraverwachsungen. Ausgedehnte bronchopneumonische Herde der rechten Lunge. Oedem der linken Lunge. Operative Magenfistel. Braune Atrophie des Herzens und der Leber. Atrophie und starke Anthrakose der Milz. Atrophie der Nieren. Adenomatöse Hypertrophie des rechten Prostatalappens. Leichte trabekuläre Hypertrophie der Harnblase. Mäßige Atherosklerose der Aorta. Fibröse Atrophie der Gaumenmandeln. Multiple Karzinoide des Ileum. Die Darmschleimhaut ist schmutziggelb, dünn; im unteren Ileam finden sich 8 kleine, reiskorn- bis erbsgroße, innerbalb der Schleimhaut sich vorwölbende feste, auf dem Durchschnitt gleichmäßig grau aussehende Erhebungen.

Mikroskopisch stellte sich das Kardiakarzinom als zerfallener Plattenepithelkrebs heraus, die Karzinoide zeigten das typische Bild.

8. Fall: Heinrich Sch. (S. N. 895. 1916), 66 Jahre alt, Landmann.

Klinische Diagnose: Prostatahypertrophie, Zystitis, Nephritis, Reitumstenose nach Radiumbehandlung.

Die Obduktion ergab: adenomatöse Hypertrophie besonders des rechten Prostatalappens mit starker Entzündung. Alte Operationsnarbe an der Sectio alta. Verwachsungen der vorderen Blasenwand sowie des Netzes im Gebiete der Operationsnarbe. Chron. papillär-hämorrhagische, schrumpfende Urozystitis. Nekroti- 
sierende eitrige chronisehe Urethritis und Zystitis mit Harngries und kleinen Harnröhrensteinchen besonders rechts. Chronisch eitrige Pyelonephritis mitSchrumpfungs: vorgängen besonders links. Perihepatitische und perisplenitische Verwachsungen. Rechtsseitiger Leistenbruch. Narben des Mesenteriums. Periappendizitische Verwachsungen. Flache, vernarbende Geschwüre des Blinddarms und Mastaarms. Blntungen und Polypen der Magenschleimhaut. Fettdurehwachsungen und kleine Schwielen der Herzmuskulatur. Mäßige Sklerose der Kranzarterien. Lungenödem. Sklerose der Aorta. Geringe der großen Halsarterien. Stauungshyperämie der Milz und Leber sowie der Luftröhren- und Rachenschleimhaut. Narben der Gaumenmandeln. Verkalkte tuberkulöse Heide der Lungen. Pleuraverwachsungen. Verwachsungen der harten Hirnhaut. Kolloidknoten der Schilddrüse. Multiple Karzinoide des Heum. Die Schleimhaut des Jejunum ist mäßig gerötet und ziemlich dünn; etwa $3,8 \mathrm{~cm}$ oberhalb der Ileozökalklappe zeigen sich in der Schleimhaut hervorragende, stecknadelkopf- bis über kirschgroße, z. T. pilzförmige und tief bis an die Serosa reichende Erhebungen, von denen dje größten in der Mitte eine kleine Einsenkung erkennen lassen und im ganzen eine ziemlich gleichmäßige Schnittfläche darbieten. Die größten sind von etwas weicherer Konsistenz; im ganzen sind 36 solche Knötchen vorhanden in einem $2,30 \mathrm{~cm}$ langen Ileumstück (s. auch Taf. XI, Fig. IV).

Mikroskopisch handolt es sich hier um ebenfalls mit ihrer Hauptmasse in der Submukosa liegende, die Muscularis muscosae auftasernde Tumoren. Sie weisen im groben und ganzen denselben Bau auf wie die vorher beschriebenen. An Masse überwiegt bier wieder die epithelähnliche Substanz. Diese ist teils strangförmig, teils in KJumpen angeordnet, unter sich zusammenhängend. Die Stränge sind solide, doch sind die Randzellen regelmäßiger und dichter gelagert, so daß man doch den Eindruck von mit losen, hier meist rundlichen Zellen gefüllten Schläuchen hat. Das Tumorgewebe wuchert bis in die Mukosa. Hier bat es die Schleimbaut teils auseinander- oder zusammengedrängt, teils scheinen die Tumorstränge in Krypten überzugehen. Die Zellen besonders der kleinsten 'Tumoren weisen z. T. Verfettung auf. Auch bier bildet, wie bei den übrigen Tumoren, der Zellkern die Hauptmasse der Zellen. Die Kerne der den Schlauch bildenden Zellen liegen hier meist im äußeren Drittel der Zellen, nicht nach dem Lumen zu. Wie bei den übrigen Tumoren ist das Stroma ziemlich kernreich, führt nicht sehr reichliche Kapillaren unb Blutgefäße.

9. Fall: Valentin R. (S. N. 160. 1917), 53 Jahre alt, Verkehrskontrolleur. R. wurde moribund mit Magenblutungen eingeliefert.

Die Obduktion ergab: großes chronisches rundes Geschwür des Magens an der kleinen Kurvatur, mit Arrosion mehrerer Arterienäste. Großer Bluterguß in den Magen. Blutiger Schleim in Speiseröhre und Dünndarm. Hochgradige allgemeine Blutarmut. Chronisehe Gastritis. Multiple, z. T. vernarbende Geschwüre des Magens. Vernarbtes Geschwür der vorderen Wand des Duodenums, grobes Divertikel an der hinteren Wand. Mageninhaltsaspiration in die Luftwege. Starkes Oedem und kleine bronchopneumonische Herde der Lungen. Chronisches Lungeneniphysem. Sklerose der Lungenarterien. Erweiterung and Hypertrophie des rechten Herzens. Reste von Aorten- und Mitralendokarditis. Starke Verfettung der Herzmuskulatur. Thrombosen und Venensteine im Plexus prostaticus. Starke 
Atherosklerose der Aorta und zahlreicher peripherer Arterien, besonders des Beckens und des Herzens. Schwielen der Herzmuskulatur. Narben und zuhlreiche Kalkkörperchen der Nieren. Nierenbeckensteine links. Leichte Prostatahypertrophie. Pleuraverwachsungen. Vernarbende und verkalkende Tuberkulose der Lungenspitzen. Ausgedehnte verkalkte Tuberkulose bronchialer und trachealer Lymphknoten. Starke Anthrakose der Lungen, der Lymphknoten des Brust- und Oberbauchraums sowie der Milz. Braune Atrophie der Leber. Perihepatitische Verwachsungen. Karzinoid des unteren Ileum. Im unteren Ileum findet sich reichlich mit Blut untermischter Schleim sowie flüssiges Blut. Die Schleimbant im ganzen von hellgrauroter Farbe. $55 \mathrm{ccm}$ oberhalb der Bauh in'schen Klappe zeigt sich eine flache, etwa kirschkerngroße, von Schleimhaut bedeckte, sich fest anfühlende Erhebung.

Mikroskopisch gibt sich die Erhebung als typisches Karzinoid mit ziemlich geringer Verfettung zu erkennen. Eine Reaktionszone in der Ungebnng der Zone ist nicht nachweisbar. Der Tumor ist in seiner Hauptmasse in der Submukosa gelagert, liegt aber der Mukosa viel dichter und inniger - ohne merklichen Uebergang - an als der Muskularis. Auf der Höbe des Tumor ist die Schleimbautschicht verdünnt und teilweise ohne Kernfärbang. An einzelnen Stellen sind zwischen beiden Muskelschichten Epitheloidzellgruppen erkennbar. An Menge überwiegt das Epithelzellengewebe das des Stroma. Ersteres besteht vielfach aus sehr regelmäßig gebauten Zylinderzellschläuchen, deren Lumen meist leer ist. An anderen Stellen sind dio Zellen zu unregelmäßigen Klumpen gesammelt. In diesem Fall sind die Zellgrenzen meist deutlicher wahrnehmbar als sonst. An den Zylinderzellen der Schläuche konnte nirgends eine auffällig vergröberte Basis nachgewiesen werden, ihre Kerne liegeu im äuberen Drittel der Schlanchwand, selten in der Mitte. Ihrer Form nach sind sie meist sehr regelmäßig kreisrund, selten länglich. Ihre Durchschnittsgröße ist gering. Doch konmen vielfach größere, bis zum doppelten Durchmesser vor.

10. Fall: Wilbelmine K. (S. N. 596. 1917), 35 Jabre alt, Arbeitersfrau.

Klinische Diagnose: Pneumonie, Lungenabszef.

Die Obduktion ergab: eitrige Tracheitis und Bronchitis. Ausgedehnte bronchopneumonische Herde, besonders des rechten Unterlappens. Pleuraverwachsungen. Abgekapseltes Empyem rechts. Lungenödem, Follikelschwellung der Milz. Perisplenitische Verdickungen und Verwachsungen. Netzverwachsungen. Stauungshyperämie der Milz nnd Nieren. Sklerose der Aorta, Mitralis und peripherer Arterien. Sehnenflecke des Epikards. Vernarbtes Magengeschwür. Perigastrische Verwachsungea. Verwachsungen der harten Hirnhant. Kleines Karzinoid des Ileum.

Die Schleimbaut des Dünodarms war glatt, gallig imbibiert. Im unteren Teil des lleum fand sich das erbsgroße weiße Knötchen, über das die Schleimhaut glatt hinwegzog.

Mikroskopisch: Das typische Bild des Karzinoid.

11. Fall: (J. N. 367 b. 1914) 16//2 Jahre alt, Schiffsjunge. Boi der Untersuchung einer nekrotisierend eitrigen Wurmfortsatzentzündung, die sich durch besondere Reichlichkeit von oxyphil gekörnten Zellen auszeichnete, fanden sich in 
einigen Gefrierschnitten in der Schleimhant und Submukosa gelegene, nur durch wenige Schnitte hindurch verfolgbare typische Karzinoidnester und -Zapfen. Im nach dem Befunde an den Gefrierschnitten sofort eingebetteten selben Stück konnten die Zapfen nur noch in drei Schnitten in der Submukosa gefunden werden.

12. Fall: (J. N. 59. 1915) $251 / 2$ Jahre alt. Appendizitis. An der Spitze findet sich ein kleines Knötchen, kaum stecknadelkopforoß. Nikroskopisch besteht es aus unregelmäßig großen, mit chromatinreichen Kernen versehenen Epithelzellen vom typischen Bau der Karzinoide. Sie liegen fast ausschließlich in der Mukosa. Hier und da sind daneben in Follikeln auch mit niedrigem zylindrischem Epithel ausgekleidete Gänge und solide Zapfen vorbanden. Bei der Färbung der Gefrierschnitte mit Scharlachhämalaun weisen die Zellen im Gegensatz zu den benachbarten normalen Drüsen ziemlich reichlich Fettröpfchen auf; auch die Zellen des z.T. recht zahlreichen Stromas enthalten Fettröpfchen. - Entfernt von dem Knötchen, das sich in den verschiedenen Schnitten aus durchschnittlich 60 bis 150 Epithelzapfen und Strängen zusammensetzt, finden sich noch drei getrennte, sebr viel kleinere Herde, die aus 3-12 Zapfen bestehen, deren Zellen übrigens auch Fettröpfohen enthalten und die z. T. in vergrößerten Follikeln liegen.

13. Fall: Peter L. (S. N. 344. 1914), 70 Jabre alt, wurde sterbend mit der Diagnose "Himblutung" in die Klinik eingelicfert. Die Sektion ergab eine sehr starke allgemeine Atherosklerose. Ausgedehnte frische und alte Hirnblutungen. Braune Herzatrophie mit starker Schwielenbildung. Hydronephrotische Nierenschrumpfung. Steine im Nierenbecken. Narben der rechten Niere. Pyelitis. Chronische Urozystitis mit Trabekularbypertrophie und Divertikeln. Steine in den Divertikeln. Umschriebene epidermoidale Umwandlung der Harnblasenschleimhant. Starke Anthrakose der Lungen, der tracheobronchialen Lymphknoten, der Leber und der Milz. Hämosiderose der Leber. Pleuraverwachsungen. Verkalkte Rippenknorpel. Arthritis doformans der Sternoklavikulargelenke. Prostatakonkretionen. Kleines, etwa erbsgrohes Karzinoid des Wurmfortsatzes.

Mikroskopisch: Der. Wurmfortsatz zeigt sich an der Stelle des Knotens in eine solide Masse verwandelt. Ein Lumen ist nicht mehr zu erkennen. Unregelmäßige Massen, Stränge, Schlänche, Gebilde aus rundlichen, polyedrischen, kubischen und zylindrischen Zellen durchsetzen den ganzen Durchschnitt, so daß als Rest des Wurmes nur ein großer Teil der Längs- und Ringmuskulatur erkennbar bleibt. Es.wechseln Stellen, wo zwischen den unregelmäßigen Zellhaufen oder auch wieder ganz regelmäbig gebildeten Zylinderzellschläuchen mit offenem Lumen nur ein ganz feines Stromanetz erkennbar ist, mit solchen, wo sich die beschriebenen Zellgruppen im Bindegewebe und Muskulatur — diese völlig auffasernd - hineinschieben. Stellenweise sind auch Gefäßwandungen derart auf das Zehnfache ibrer normalen Dicke verbreitert. An einer Stelle liegen Epitheloidzellon streifig, wie $Z$ wiebelschalen, um ein kleines Gefäß angeordnet, am Außenrande der Längsmuskulatur unter der Serosa.

Stelle ich die wichtigsten Daten dieser 13 Fälle zusammen, so ergibt sich folgendes: Es handelt sich um 10 Fälle von lleum- und 3 von Appendixkarzinoid; erstere betreffen 6 Männer im Alter von 53-70 Jahren (Mittel 63,2) und 4 Frauen im Alter von 20-38 Jahren (Mittel 31), also 
ein Gesamtmittel von 47,1 Jahren. Rechne ich dazu noch 3 weitere später von Lubarsch beobachtete Fälle (17, 67 und 49 Jahre) dazu, so beträgt das Durchschnittsalter 46,5 Jahre. Die 3 Appendixkarzinoide betreffen dagegen ausschließlich Männer im Alter von $16 \frac{1}{2}, 25 \frac{1}{2}$ und 70 Jahren, so daß hier das Durchschnittsalter 37,3 Jahre beträgt; rechne ich hierzu noch 2 ältere, bisher noch nicht veröffentlichte und anch von mir nicht beschriebene Fälle von Lubarsch (17 jähriger Jïngling und 26 jährige Frau), so komme ich sogar zu einem Durchschnittsalter von 31 Jahren. Es bleibt daher in den beschriebenen Fällen von Ileumkarzinoid nur bei den Männern die Regel bestehen, daß das Karzinoid in einem Alter jenseits des durchschnittlichen . Karzinomalters auftritt, während hier bei den Frauen das Vorkommen unter dem gewöhnlichen Karzinomalter liegt, so daß wir uns im Gesamtmittel diesem wieder ganz nahe befinden. Ein Vergleich mit der Literatur ergibt, daß in den veröffentlichten Fällen das Durchschnittsalter etwa bei 55 Jahren liegt und zwischen Frauen und Männern hierin kein erheblicher Unterschied besteht. Dagegen liegt das Durchschnittsalter bei Appendixkarzinoiden erheblich niedriger, wie auch bei unseren Fällen. (Vergl. auch Zaaijer, Beiträge f. klin. Chir. 1907. Bd. 54. H. 2.) Ein besonderer Zusammenhang mit Beruf bzw. Lebensweise scheint nicht vorzuliegen, ebensowenig mit andersartigen Erkrankungen. Dagegen stellt sich die Vergesellschaftung mit anderen Tumoren folgendermaßen dar:

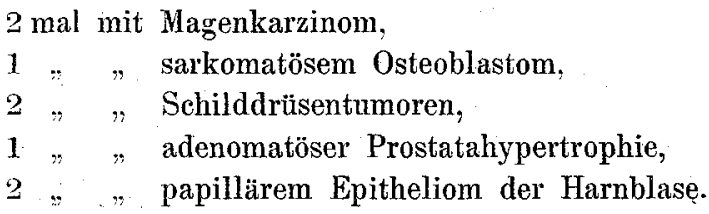

Daneben ist zu bemerken, daß, abgesehen vom Sarkom bei einer Frau, in allen Fällen mit dem Vorhandensein eines andersartigen Tumors die Karzinoide multipel vorkamen und es sich um Männer handelte. — Ein häufigeres Zusammentreffen besonders mit Magenkarzinom ist mir auch in der mir zugänglichen Literatur aufgefallen. - Unter Einrechnung der späteren 3 Fälle handelte es sich 4 mal um Einzelknoten, 8 mal multiple. Darunter werden einmal auf einem 2,30 m langen Ileumstiick $36 \mathrm{Knötchen} \mathrm{gezählt,}$ in einem anderen Falle 30. Ihre Größe schwankt zwischen Stecknadelkopf- und Kirschgröße. In histologischer Hinsicht ist zunächst eine gewisse Einförmigkeit der Bilder hervorgetreten. Die Tumoren zeigen einen ziemlich gleichmäßigen organartigen Aufbau. Regelmäßig gebaute Schläuche kommen vor, sind aber seltener als solide Stränge und Zapfen und stehen meist wie diese im Zusammenhang miteinander. Die Zellgrenzen sind oft unscharf, Zellen und Kerne verhältnismäßig klein, dadurch dichtliegend erscheinend und in der Form dadurch nicht aus- 
gesprochen epithelial, besonders im Vergleich zu typischen Karzinomen. Der Kern išt im Verhältnis zur gesamten Zellmasse groß. Was Form der Zelle anbetrifft, konnte keine regelmälig vorkommende Besonderheit gegenüber ähnlichen anderen Tumoren gefunden, ebensowenig konnte die allerdings stellenweise beobachtete Lagerung der Zellkerne in den Schläuchen nach der Mitte oder gar dem freien Rande der Zellen zu regelmäßig beobachtet werden. Die Kernform war meistenteils rund, besonders in den Schläuchen, seltener oval oder länglich.

Wenden wir uns jetzt, nach der Schilderung und Zusammenfassung unserer Befunde, zu den hauptsächlichsten Streitfragen über diese eigenartigen Neubildungen, so können wir uns zunächst am leichtesten abfinden mit den Ansichten Oberndorfer's, Milner's und Saltykow's. - Daß es sich um endotheliale Neubildungen auf entzündlicher (Milner) oder nicht entzündlicher Basis handle, ist eine Annahme, die fast ganz verlassen ist, in allen späteren Untersuchungen und so auch meinen Befunden genïgende Stütze nicht findet. Sie ist wohl auch nur dadurch entstanden, daß; wie ähnlich von mir hervorgehoben werden muBte, der epitheliale Charakter der Zellen hinsichtlich der. Form oft verwischt ist, d. h. die Zellen sind häufig vorwiegend rundlich und nicht ausgesprochen zylindrisch oder kubisch: Das ist aber etwas, was man überall in epithelialen Neubildungen findet, wenn die Zellen auf begrenztem Raum dicht zusammengedrängt werden; wo sie weniger dicht gedrängt sind, besonders dort, wo sie röhren- und drüsen- oder netzförmige Anordnung zeigen, tritt die Form zylindrischer Epithelien dentlich hervor, besonders auch in. den seltenen Fällen, wo in Follikeln Epithelstränge liegen. Dab die Hohlräume, in denen die Zellen liegen, 'keine Lymphräume sind, das ergibt sich einmal daraus, daß keine Beziehungen zu den Blutgefaßen sich finden, und weiter aus dem Mangel einer besonderen endothelialen Deckzellenauskleidung: andererseits ergibt sich aus dem, wenn auch nicht immer nachweisbaren Zusammenhang mit Lieberkühn schen Krypten positiv' die Beziehung zu den drüsigen Bestandteilen des Darmes. Ferner besteht keine Aehnlichkeit mit den Bildern, wie sie z. B. von Baumgarten als Lymphangitis hyperplastica bei entzündlichen Darmerkrankungen beschrieben sind, wo die Kerne verhältnismäßig kleiner, der Zelleib gequollen, das Protoplasma heller ist und niemals große rundliche oder verzweigte Zapfen gebildet werden. ---

Ebenso scheint mir auch die Annahme Oberndorfer's, daß es sich um den Nebenpankreasanlagen nahestehende Fehlbildungen, oder Saltykow:s, daß es sich um Langerhans'sche Inselbildende Pankreaskeime handelt, nicht genügend begründet zu sein. ln dieser Hinsicht sei nur auf folgendes hingewiesen: Eine Aehnlichkeit mit Langerhans'schen Inseln ist bezüglich der einzelnen Zellen in oberflächlicher Weise insoweit 
vorhanden, als sie meist nicht ausgesprochen zylindrisch, sondern mehr rundlich oder eckig sind; dagegen besteht schon nicht die gleiche auffallende Helligkeit des Protoplasmas; vor allem fehlt aber der für die Langerhans'schen Inseln so kennzeichnende Gefäßreichtum vollständig. Ebensowenig kaun von einer Aehnlichkeit mit Nebenpankreasanlagen die Rede sein. Sowohl bei denen des Magens wie denen des Darms - und ebenso auch bei den aus ihnen herrorgehenden Adenomyomen dieser Organe - kann man, wie aus zahlreichen mir von Herrn Geh. Rat Lubarsch zur Verfügung gestellten Präparaten hervorgeht, in der Hauptsache zwei Arten unterscheiden: 1. solche, in denen typisches Pankreasgewebe vorhanden ist, 2. solche, in denen nur den Pankreasausführungsgängen entsprechende, mit hohem zylindrischem Epithel ausgekleidete, meist vielfach verästelte und oft zystisch erweiterte Röhren sich finden. $\mathrm{DaB}$ in beiden Arten mitunter auch weniger charakteristische Abschnitte - vielleicht auch sogar solide Epithelstränge, wie sie Lubarsch allerdings nicht fand -- vorkommen, ist natürlich nicht von entscheidender Bedeutung, denn selbst diese Abschnitte stimmen nicht mit dem überein, was die Karzinoide durchgängig zeigen. Ich muß also in allen Punkten auf Grund meiner Befunde mich dem anschließen, was Burkhardt gegen Oberndorfer und Saltykow bereits ausgeführt hat. Ebensowenig kommi wohl noch die Ansicht in Betracht, daß es sich um den Drüisenpolypen des Darmes gleichzustellende Gebilde handelt. Selbst Ribbert, der an entschiedensten die Ansicht vertritt, daß alle Darmpolypen angeborene aus Entwicklungsstörungen entstandene Bildungen sind (s. „Das Karzinom des Menschen". S. 165), vergleicht die Dünndarm- und Wurmfortsatzkarzinoide nicht mit den Darmpolypen. Der Unterschied liegt ja auch allein schon darin auf der Hand, dab die Polypen offene, mit hohem, einschichtigem oder allenfalls auch mehrschichtigem' Epithel ausgekleidete Schläuche bilden, - die Karzinoide dagegen fast ausschließlich aus soliden, von niedrigen Zellen gebildeten Strängen und Zapfen bestehen. -

Wenden wir uns nun zu der Hauptfrage - 1. ob wir es überhaupt mit echten Blastomen oder nur mit Hamartien und Choristien (Albrecht), Gewebsmißbildungen (Lubarsch) zu tun haben, 2. ob, falls es sich um echte Gewächse handelt, sie als echte Krebse angesehen werden dürfen oder nicht. Die erste Frage kann gut nur beantwortet werden, wenn man die Karzinoide mit zweifellos einfachen epithelialen Gewebsmißbildungen des Darmes vergleicht. Unsere Kenntnisse darüber sind nun nicht sehr grob. Lubarsch ist wohl der Einzige, der auf diesem Gebiete wenigstens einige systematische Untersuchungen besonders am Wurmfortsatz und Dünndarm vorgenommen hat. Nach seinen Angaben (Verhandl. d. deutsch. Pathol. Ges. Stuttgart 1906 und Verhandl. d. Internat. Med. Kongr. in Budapest 1909) sind epitheliale Entwicklungsstörungen im Dünndarm and 
Wurmfortsatz außerordentlich selten; das Einzige, was er bei Föten, Neugeborenen und Säuglingen in dieser Hinsicht finden konnte, waren Drüsenund Follikelheterotopien, d. h. submukös gelagerte Follikel und Krypten, wobei hier und da die Drüsen in den Follikeln lagen, wie es schon vorher gelegentlich auch von 0 rth bei jugendlichen und älteren Individuen beobachtet worden war. Aber diese Driisenheterotopien haben wenigstens nach allem, was bisher an Föten, Neugeborenen, Säuglingen, Kindern und Erwachsenen beobachtet ist, immer den Typ mehr oder weniger vollständig ausgebildeter Li eberkühn'scher Krypten, stets Drüsencharakter und zeigen weder netzförmige noch solide Stränge und Zaplen. - Wir kommen daher zu dem Ergebnis, daß es sich um einfache Gewebsmißbildungen nicht handelt, nicht einmal in den Fällen der allerkleinsten Karzinoide. Damit ist aber keineswegs gesagt, daß sie nicht doch in Beziehungen zu Gewebsmißbildungen stehen, im Gegenteil tritt aus einem Teil unserer Fälle (bes. Fall 12) die Beziehung zu den einfachen angeborenen Driisenheterotopien aus ihrem Liegenbleiben in Follikeln deutlichst hervor, eine Beobachtung, die Lubarsch schon früher in einem Falle eines erbsgroßen Karzinoids des Wurmfortsatzes bei einem 17 jährigen Jüngling gemacht hatte, wo er an Stellen, die entïernt von der Neubildung lagen, submukös gelegene Follikel und Drüsen ' fand (Verhandl. d. deutsch. Pathol. Ges. Bd. 10. S. 213). Es kann daher wohl als gesichert gelten, dab die Karzinoide in Beziehungen zu der häufigsten Art embryonaler Gewebsmißbildungen im Darm stehen. Nun wissen wir durch die Untersuchungen von Rob. Meyer (Lubarsch-0 stertag. Ergebnisse: Jahrg. 15 II) und Lubarsch (Verhandl. d: internat. med Kongr. in Budapest 1909), daß die verschiedenen embry0nalen Entwicklungsstörungen für die Entstehung von Gewächsen von sehr verschiedener Bedeutung sind, und daß auch die Schicksale der embryonalen Gewebsmißbildungen verschiedenartig sein können. Rob. Meyer und Lubarsch unterscheiden, unbeschadet einiger Abweichungen im einzelnen, in der Hauptsache drei Arten von embryonalen Entwicklungsstörungen, nämlich 1. abnormes Bestehenbleiben von Organen und Geweben ans dem Embryonalleben, 2. embryonale Dysplasien, 3. Differenzierungshemmungen und Verlagerungen. Von diesen drei Arten besitzen die beiden ersten die geringste Bedeutung für die Blastombildung. Man wird also zunächst zu fragen haben, zu welcher Gruppe von Entwicklungsstörungen denn die in Verbindung mit Karzinoiden beobachteten embryonalen Gewebsmißbildungen' gehören. Hier könnte man zunächst geneigt sein, sie ohne weiteres in die dritte Gruppe - Verlagerungen -- einzureihen, wofür ja auch besonders der Umstand geltend gemacht werden kann, daß ein großer Teil der Karzinoide sich in erster Linie submukös entwickelt. Aber so ganz sicher ist diese Annahme doch nicht, da nach den entwicklungsgeschichtlichen Untersuchungen von Retterer und Stöhr die Bildung der 
Lieberkühn'schen Krypten submukös und in enger Verbindung mit den Follikeln erfolgt. Die Befunde submuköser Follikel und submuköser extraoder intrafollikulärer Drüsen würde somit keine Verlagerung, sondern nur ein abnormes,Verbleiben am ursprünglichen Bildungsort bedeuten, und das könnte vielleicht zur Erklärung der auffallend geringen Wachstumsgeschwindigkeit der Karzinoide verwendet werden können. Bezüglich der Schicksale der entwicklungsgeschichtlichen Gewebsanomalien unterscheidet Lubarsch vier Möglichkeiten: 1. Untergang oder wenigstens Rückbildung, 2. Stehenbleiben auf embryonaler Stufe, 3. synchrones Wachstum, 4. unabhängiges blastomatöses Wachstum. Von diesen vier Möglichkeiten können wir die beiden ersten für die ausgesprochenen - wenn auch noch so kleinen Karzinoide - ausschließen. Aber auch die dritte kommt kaum in Betracht. Das zeigt der Vergleich mit Nebenpankreasanlagen, sogenannten Adenomyomen, und größeren submukösen Follikel- und Drüsenbildungen bei Erwachsenen. In allen Fallen, wo wir ein einfaches synchrones Wachstum annehmen müssen, besteht eine einfache Vergrößerung (oder allenfalls auch Vermehrung) der drüsigen Bestandteile, genau wie beim physiologischen Wachstum überhaupt, ohne Aenderung des Zell- and Gewebscharakters. Das ist ja aber gerade bei den Karzinoiden das Auffallende, daß der Zelltypus sich von dem der Epithelien - und zwar nicht nur der fertigen, sondern auch der embryonalen (weswegen also auch kein Stehenbleiben auf embryonaler Stufe in Betracht kommt) - wesentlich unterscheidet. Ferner ist ein einfaches synchronisches Wachstum auch deswegen auszuschlieben, weil auch die Drüsenform nicht gewahrt ist, und die Vergrößerung weit über das hinausgeht, was wir bei einfacher Wachstumsvergrößerung zu sehen bekommen. Der Vergleich der einfachen drüsigen Gewebsmißbildungen des Darms bei Föten, Kindern und Erwachsenen mit den Karzinoiden vielmehr zeigt, das hier eine ganz selbständige und weit über das physiologische Maß hinausgehende Vergrößerung und Vermehrung vorliegt: Es muß daher als durchaus gesichert angesehen werden, daß wir es mit echten selbständigen Blastomen, also nicht einfachen Hamartien oder Choristien, sondern Hamarto- und Choristoblastomen zu tun haben. -

Somit kommen wir zu der zweiten, am meisten umstrittenen Frage, ob es sich um echte Krebse handelt oder nicht. Hier ist ja zunächst durchaus zužugeben, daß erhebliche Unterschiede zwischen den Karzinoiden und den gewöhnlichen Darmkrebsen bestehen, Unterschiede, die ja anch Burckhardt scharf betont hat: Ihr vorwiegend multiples Auftreten, ihre meist recht geringe GröBe, ihr abgeschlossener organartiger Bau, ihre (zum mindesten relative) Gutartigkeit, ihr histologisches Verhalten (fast rein solider Bau, Kleinheit der Zellen, Fehlen der Schleimbildung und Kernteilungen; spärliches und meist wenig entzündetes Stroma). Daß diese 
Unterschiede aber nicht völlig überzeugend und ausschlaggebend sind, ergibt sich allein schon aus der verschiedenen Stellungnahme der einzelnen Autoren, wie ja Burkhardt, Versé, Lubarsch (Verhandl. d. deutsch. Pathol. Ges. Bd. 12. S. 39 u. 40) und Ribbert („Das Karzinom des Menschen". S. 186 u. 187) an der krebsigen Natur dieser Neubildungen festhalten. Versé und Burkhardt haben in dieser Hinsicht besonders betont, daß die Karzinoide auch Metastasen machen können, und entsprechende Fälle beschrieben. Auch ich bin in der Lage einen derartigen Fall anführen zu können:

14. Fall: Richard S. (S. N. 36. 1917), 43 Jahre alt, Arbeiter.

Klinische Diagnose: Darmkrebs mit Metastasen.

Anatomische Diagnose: Kleiner infiltrierender Krebs desWurm. fortsatzes, ausgedehnte Metastasen des Netzes, Mesenteriums, des Zwerchfells, der Darm- und Magenserosa, der retroperitonealen, periportalen, peripankreatischen und mesenterialen Jymphknoten. Lahlreiche und sehr große Metastasen der Leber. Einwachern von. Lymphknoten- und Gekrösemetastasen in den Ductus choledochus, Magen, Milz, Dünn- und Dickdarm. Große Metastasen am Beckenboden. Fibrinös-hämorrhagische Peritonitis. Laparotomiewunde. Leichte Atraphie der Milz, Leber, Schilddrüse und Nebennieren. Thrombose des Plexus prostaticus. Frische Thromben der Pfortader. Atrophie der Gaumenmandel und Darmschleimbaut, Zyste des Nebenhodens. Verfettung der Herzmuskulatur, geringe Shlerose der Mitralis und Aorta. Katarrhalisch-eitrige Tracheitis und Bronchitis. Oeirem und bronchopneumonische Herde beider Lungen. Aus dem Leichenbefund führe ich nur den Befund am Darm an: Die Dünndarmschleimhaut ist im ganzen von hellroter Farbe, stellenweise auch mehr gelblich, mit im allgemeinen wenig hervortretenden Falten und Follikeln. Auch ist sie im ganzen dünn. An mohreren Stellen wölben sich in ihr vom Mesenterium her bis kirschkerngroße, in der Mitte eingezogene, gelbliche Knoten vor. Im unteren Teil des Ileum ist die Schleimhaut stärker gerötet. Dicht an der Mleozökalklappe wölbt sich ein noch von Schleimhaut überzogener etwas höckriger Knoten vor. Der Wurmfortsatz ist kurz, nur $6 \mathrm{~cm}$ lang, an der Spitze stark verdickt und verengt. Serosa und Muskulatur grenzen sich auf dem Querschnitt scharf ab, während Submukosa und Schleimhaut in eine gelblichweiße, gleichmäßige Tumormasse umgewandelt ist. An der äußersten Spitze ist im übrigen der Wurmfortsatz vollkommen frei. Der Dickdarm ist im ganzen weit. Die Schleimhaut gefaltet, hellrötlich, mit vereinzelten, kirschgrofen, rom Mesenterium sich vorwölbenden Knoten.

Vom mikroskopischen Befund seien zunächst die Uebereinstimmungen mit den Karzinoiden hervorgehoben: Wie bei diesen ist die Wuchernng ziemlich begrenzt und erstreckt sich sowohl auf Schleimhaut wie Submukosa, nur mit kleinen Ausläuferm auch bis in die Muskularis. Die Schleimhaut ist in dem Gebiet stark verdünt, Follikel und Drüsen fehlen und sind durch die epitheliale Wucherung ersetzt. Diese tritt so- 
wohl in Form solider Stränge und Zapfen als auch größerer Alveolen und netzförmig durchbrochener Bildungen auf; das Stroma ist im allgemeinen spärlich und wenig zellreich und nur an der Grenze zur Muskulatur reichlicher von Rundzellen durchsetzt. Abweichend von den typischen Karzinoiden ist folgendes: Es finden sich etwas reichlicher einfache Epithelschläuche, reichlicher Kernteilungsfiguren, wenigstens in den dicht an der Grenze zur Muskulatur und in ihr gelegenen Teilen; die Epithelien selbst sind vielfach etwas höher, z. T. sogar ausgesprochen zylindrisch, teilweise leicht hydropisch gequollen und dann das Protoplasma heller. Die Kerne selbst von mehr wechselndem Chromatingehalt. Ueberhaupt besteht nicht solche Einförmigkeit wie in den kleineren Karzinoiden. - Es liegt aber auf der Hand, daß diese Unterschiede nicht von grundsätzlicher Bedeutung sind, sondern einfach die Folge des rascheren Wachstums; wie wir ja ganz allgemein alle rascher wachsenden Geschwülste durch eine mehr oder weniger erhebliche Vielgestaltigkeit der Zellen und Kerne sich auszeichnen sehen, und wie auch in den Metastasen des Falles sowohl Vielgestaltigkeit als Kernteilungen und regressive Veränderungen einen weit höheren Grad erreichen als im Primärkrebs. Viel wichtiger scheinen mir die Uebereinstimmungen - die erhebliche Kleinheit des Wurmfortsatzkrebses trotz der gewaltigen Metastasenbildung, die Uebereinstimmungen in Anordnung und Ausbreitung (fast ganz auf Submukosa und Schleimhaut beschränkt), die, wenn auch nicht durchgängig aber doch vielfach vorhandene Uebereinstimmung des Zellcharakters, das Verhalten des Stromas.

Zeigt somit auch dieser Fall wieder, daß es sehr kleine Wurmfortsat $\dot{z}$ krebse gibt, die in der Hauptsache dem Typus der Karzinoide entsprechen und sich doch durch gewaltiges destruierendes Wachstum und Metastasenbildung (die Kennzeichen der Bösartigkeit) auszeichnen, so ist damit allein die. Frage nach eder Natur der Karzinoide noch nicht erledigt. Denn es könnte ja sehr wohl ein ähnliches Verhältnis bestehen wie zwischen melanotischen Nävis und Melanokarzinomen, daß es sich um ursprünglich typisch wachsende Neubildungen handelt, die nur eine verhältnismäßig große Neigung besitzen in Krebs überzugehen - eine Anschauung, die ja von Aschoff vertreten wird. Wir müssen deswegen noch untersuchen, welche Wachstumsverhältnisse denn die kleinen und kleinsten Karzinoide darbieten und wie wir uns ihre Entwicklung vorstellen müssen: In dieser Hinsicht muß zunächst hervorgehoben werden, daß sie sich zweifellos in erster Linie submukös entwickeln, und dab von hier aus ihre Ausbreitung. nach den verschiedenen Richtungen hin erfolgt. Fall 1, 2, 3, 5, 8 (Vergl. die Textfiguren) zeigen diese Verhältnisse am reinsten und beweisen am deutlichsten, daß das Wachstumszentrum in der Submukosa sich findet. Von hier aus erfolgt selbst in den kleineren Neubildungen ein Wachstum, das nicht einfach expansiv und verdrängend, sondern deutlich infiltrativ 
und oft genug auch ausgesprochen diskontinuierlich ist (s. die Skizzen 1 und 2, 4 und 5); hier hat man Bilder, die gar nicht gut anders als im Sinne einer destruierenden Ausbreitung, ganz wie bei echten Krebsen, gedeutet werden können. Auf diese Bilder lege ich den allergrößten Wert; denn auf das Vorkommen der epithelialen Zapfen in Submukosa and Muskularis allein kann ja noch nicht anf ein destruierendes Wachstum geschlossen werden, wenn man zu der Annahme gezwungen ist, daß die Bildungen mit, abnorm gelagerten Drüsen in Zusammenhang stehen; aber die Ausbreitung von einem noch umschrieben groben Mittelpunkt in der Submukosa nach allen Richtungen hin durch kleine Stränge und Zapfen, wie es sich in fast allen Fällen nachweisen läßt, zeigt doch aufs Deutlichste, daß die primär außerhalb der Schleimliaut entstandene. Wucherung nicht an diesem Ort haltmacht, sondern über die normalen Schranken hinaus schleichend und zerstörend wächst. Man kann auch nicht gut versuchen, diese Bilder etwa in dem Sinne zu deuten, dab es sich um verschiedene von vornherein getrennte und erst nachträglich mit einander in Verbindung tretende Geschwulstkeime handelte, daß also etwa embryonale Drüsenverlagerungen in Schleimhaut, Submukosa and Muskularis primär vorhanden wären, die nun gleichzeitig oder nacheinander in Wucherung gerieten. Diese Annahme wird widerlegt 1. durch den Umstand, daß die bisher bekannten Drüsenabnormitäten im Darm sich nur submukös finden. 2. durch die Form der in Muskulatur, Schleimhaut und mitunter selbst Subserosa vorhandenen Geschwulstteile, die im Gegensatz zu denen in der Submukosa nicht abgeschlossene Knoten, sondern in die Gewebsspalten sich verbreitende Ausläufer, Züge und Stränge bilden. -

Ich muß daher anf Grund meiner Befunde an Dünndarm- und Wurmfortsatzkarzinoiden zu dem Ergebnis kommen, daß auch die kleinen Kankroide grundsätzlich in ihrem Wachstum mit Krebsen übereinstimmen; das hindert natürlich nicht, ihnen trotzdem eine gewisse Sonderstellung zuzumessen, die sie aber mit Krebsen auch anderer Gegenden - der Haut und Schleimhäute - teilen. Lubarsch (Verhandl. d. deutsch. Pathol. Ges. Bd. 12. S. 40 u. 41) hat ausgeführt, daß wir bei den Krebsen der Haut und der meisten Schleimhäute - insbesondere des Verdauungstraktus - zwei große Gruppen von Krebsen nnterscheiden müssen: 1. solche, die von normal gelagertem, unverändertem oder bereits vorher pathologischem Epithel ihren Ausgang nehmen, und 2. solche, die von heterotopem Epithel aus sich entwickeln. Die letzteren, intra- und submuköse, kutane und subkutane Krebse (Koriumkrebse), sollen sich von der ersten Gruppe unterscheiden: 1. durch das meist völlige Fehlen einer zelligen Veränderung des Bindegewebes der Nachbarschaft (und auch des Stromas selbst), 2. durch die sehr viel stärkeren Abweichungen im Bau der Epithelien, 3. daB sie besonders gerne in Form des retikulären (oder soliden) Krebses auftreten 
und weder im Darm noch Magen jemals hoch adenomatösen Charakter annehmen. Besonders deutlich ist bei ihnen, daß das Wachstum ausschließlich von innen heraus und nicht durch Einbeziehung der Nachbarschaft erfolgt, wie eine Verbindung mit dem Schleimhautepithel oft überhaupt nicht eintritt. Man brancht hierüber nur unsere Befunde und die Burkhardt's $u$. a. zul vergleichen, um eine vollige Uebereinstimmung zu finden. Man Kann der Kennzeichnung der ron heterotopen Epithelien aus sich entwickelnden Krebse vielleicht noch anfügen, daß sie sich von der anderen Gruppe auch durch erheblich langsameres Wachstum (relative Gutartigkeit) auszeichnen. Lubarsch selbst ist hierauf schon näher eingegangen und hat betont, daß die Wachstumsgeschwindigkeit der Krebse sehr verschieden ist, dal oft genug Wachstumsstillstände vorkommen, die Vergrößerung nicht kontinuierlich, sondern schub- und stadienweise erfolgt und wir sogar spontane Riickbildungs- und Heilungsvorgänge an ihnen kennen (a. a. 0. S. 38), und weiter unten (S. 39) hebt er nochmals herror, daß es eine außerordentliche Latenz der Karzinome gibt und wir ja auch bei der Uebertragung fertiger Krebszellen eine recht lange Latenzperiode beobachten. - Alles das scheint mir die Besonderheiten der Karzinoide zu erklären: Es sind ron heterotopen Epithelien aus entstandene Neubildungen, deren Wachstumsart grundsätzlich mit der der Krebse übereinstimmt; die aber eine ungewöhnlich lange Latenzperiode besitzen und meist in verschiedenen Stadien der Latenzzeit angetroffen werden. Woraul diese auffallend lange Latenzperiode beruht, darüber kann man nur rorsichtige Vermutungen anstellen. Es wurde oben schon angeführt, dafi von Bedeutung sein kann der Umstand, daßs sie nicht von eigentlichen embryonalen Verlagerungen, sondern von embryonalen Gewebspersistenzen. aus sich entwickeln; man muß aber aluch daran denken, daß sie infolge ihrer geschützten Lage andauemden Reizen und Schädigungen in weit geringerem Maße ausgesetz sind als die von normal gelagerten Zellen ausgehenden, an der Oberfläche sich entwickelnden Krebse. Wenn man demnach auch die Karzinoide grundsätzlich den Krebsen zuzurechnen hat, so muß man doch sicher eine besondere Stellung einnehmen hinsichtlich ihrer prognostischen Beurteilung. Hier wird man fast immer die Voraussage viel günstiger stellen dürfen als bei den sonstigen Darmkrebsen. Das gilt besonders fïr die Karzinoide des Wurmfortsatzes, wo sich z. B. in den von Lubarsch beobachteten Fällen, in denen der Wurmfortsatz operativ entfernt war, seit dem Jahre 1907 und 1909 keine Krankheitsbeschwerden mehr eingestellt haben. Und auch in den von uns mitgeteilten Fällen (Nr. 11-13), die nunmehr auch fast drei Jahre zuriickliegen, sind bisher keine Anzeichen dafür vorhanden, daß die entlemten Gewächse bösartig im landläufigen Sinne gewesen wären. 
426 Hagemann, Beziehungen d. sog, Karzinoide d. Darms zu d. Darmkrebsen.

Die vorliegende Arbeit ist unter der gütigen Anleitung des Herrn Geheimrats Prof. Dr. Lubarsch verfaßt; die in derselben bearbeiteten Fälle kamen sämtlich im pathologischen Institut der Universität Kiel zur Untersuchung.

Es ist mir eine angenehme Pflicht, Herrn Geheimrat Professor Dr. Lubarsch für die Anregung zu der Arbeit, ihre Durchsicht, sowie für die gütige Ueberlassung des Materials meinen ehrerbietigsten Dank auszusprechen. 